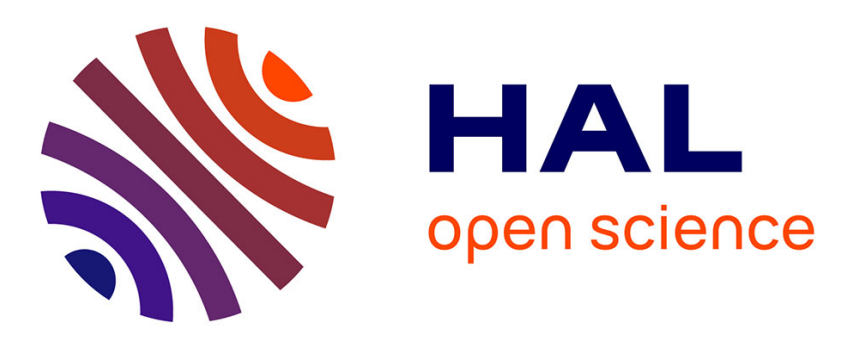

\title{
Electronically Reconfigurable Dipole Antenna Using Integrated Passive Non-Volatile Solid-State Metal-Insulator-Metal Switches
}

Methapettyparambu Purushothama Jayakrishnan, Etienne Perret, Arnaud Vena, Brice Sorli

\section{To cite this version:}

Methapettyparambu Purushothama Jayakrishnan, Etienne Perret, Arnaud Vena, Brice Sorli. Electronically Reconfigurable Dipole Antenna Using Integrated Passive Non-Volatile Solid-State MetalInsulator-Metal Switches. 2019 IEEE International Symposium on Antennas and Propagation and USNC-URSI Radio Science Meeting, Jul 2019, Atlanta, United States. pp.303-304, 10.1109/APUSNCURSINRSM.2019.8888967 . hal-02429397

\section{HAL Id: hal-02429397 https://hal.science/hal-02429397}

Submitted on 13 Jan 2020

HAL is a multi-disciplinary open access archive for the deposit and dissemination of scientific research documents, whether they are published or not. The documents may come from teaching and research institutions in France or abroad, or from public or private research centers.
L'archive ouverte pluridisciplinaire HAL, est destinée au dépôt et à la diffusion de documents scientifiques de niveau recherche, publiés ou non, émanant des établissements d'enseignement et de recherche français ou étrangers, des laboratoires publics ou privés. 


\section{Electronically Reconfigurable Dipole Antenna Using Integrated Passive Non-Volatile Solid-State Metal- Insulator-Metal Switches}

\author{
M.P. Jayakrishnan ${ }^{1}$ and Etienne Perret ${ }^{1,2}$ \\ ${ }^{1}$ Univ. Grenoble Alpes, Grenoble INP*, \\ LCIS, 26000 Valence, France \\ *Institute of Engineering Univ. Grenoble Alpes \\ jayakrishnan.mp@lcis.grenoble-inp.fr, \\ etienne.perret@1cis.grenoble-inp.fr
}

\author{
Arnaud Vena ${ }^{3}$ and Brice Sorli ${ }^{3}$ \\ ${ }^{3}$ Institut d'Electronique et Systèmes, \\ Université de Montpellier 2, 34095 Montpellier, France \\ ${ }^{2}$ Institut Universitaire de France, 75005 Paris, France \\ arnaud.vena@umontpellier.fr, \\ brice.sorli@ies.univ-montp2.fr
}

\begin{abstract}
In this article, we present for the first time, the design and experimental results of an electronically reconfigurable dipole antenna using passive and non-volatile solid state Metal-Insulator-Metal (MIM) switches. MIM switch is integrated and fabricated inclusively with the antenna, in an ambient lab environment and notably without the use of any 'clean room' facilities. Radiation pattern of the antenna could be configured electronically using DC pulses applied to the MIM switch. A $180^{\circ}$ steering of the $E$ and $H$ plane radiation patterns from positive to negative end-fire direction and vice versa is achieved by choosing $\operatorname{Set}(\mathrm{ON}) / \operatorname{Reset}(\mathrm{OFF})$ state of the switch. The antenna shows good return loss characteristics for all switch states around $2.23 \mathrm{GHz}$ and has a usable $-10 \mathrm{~dB}$ band width of $54 \mathrm{MHz}$ in common for both switch states.
\end{abstract}

Keywords: Conductive Bridging Random Access Memory (CBRAM), Metal-Insulator-Metal (MIM) Switch, Non Volatile RF Switch, Electronically Reconfigurable Antennas.

\section{INTRODUCTION}

Electronic pattern agility or reconfigurability improves efficiency and quality of a communication link to a great extent. Genius prototypes has been reported in this field, with emphasis on multi direction beam forming [1], [2]. However in most cases, active and volatile switching devices like PIN or Varactor diodes are used to achieve the reconfigurability. These devices exhibit excellent results, despite this, they need a persistent power supply to maintain their state, which adds to the power budget and limits their use in low power stand-alone devices. Moreover these switches are in form of lumped components and are generally not so compatible with the new trend of printed flexible electronics and needs to be integrated into the circuit by methods like soldering.

In this article, for the first time, we present a proof of concept of using Metal-Insulator-Metal (MIM) switches for pattern reconfigurable antenna application. MIM switches are non-volatile and passive solid state switches, derived from the concept of Conductive Bridging Random Access Memory (CBRAM) technology [3]. An MIM cell is similar in geometry to a parallel plate capacitor with one of its electrode as an electrochemically active ion-donor metal like silver or copper. The other electrode is a relatively inert ion-acceptor like aluminium or gold (see Fig.1). Electrolyte of the capacitor is

This project has received funding from the European Research Council (ERC) under the European Union's Horizon 2020 research and innovation program (grant No 772539). This work was also supported by Univ. Grenoble Alpes, and by the Institut Universitaire de France. Authors also acknowledge the HERMES platform of IES for measurements. replaced by an ion-conductor like chalcogenide glass or polymers like PMMA or Nafion. Upon application of a positive voltage with respect to active electrode, ions from the active electrode would migrate through the ion conductor to form a metallic filament which touches the inert electrode, to mark the set or $\mathrm{ON}$ state of the switch. A similar negative voltage could be used to reverse the process and break the filament to form the reset or OFF state. Switch states thus formed are non-volatile and do not require a power supply to maintain them, unless for a change of state. This process is repeatable for many programming cycles. A method is optimized for fabrication of such switches integrated into transmission lines and microwave devices on classic as well as flexible substrates. Using normal laboratory facilities, close to an industrial setup and without using any 'clean room' equipments [4], [5]. This process is potentially compatible with printing processes. Here we present the experimental results of a dipole antenna loaded with an MIM switch (using copperNafion-aluminium switching layers) integrated into a parasitic element to achieve pattern reconfigurability, using the above mentioned process, and without any soldering.

\section{Proposed Methodology}

We propose a novel idea of integrating a MIM switch into a parasitic element loaded to a half wave dipole antenna. MIM switch is added to a parasitic element (PE) in parallel with the dipole, at the centre, parallel to feed centre of dipole antenna. The width of PE is tapered to reduce the effective capacitance due to overlapping sections. Fabrication of the device is done similar to as reported in [4]. Fig. 1 shows the topology and geometry of the antenna arrangement. Dipole antenna is fed using a well-known microstrip-to slot-line balun [6]. This design is initially simulated using CST microwave studio and the results are in perfect agreement with the experiment, but are omitted from this article due to space constrains. In simulation, MIM switch is operated using a lumped resistor with values similar to filament resistance obtained in experiment [4]. We could observe that the PE acts as a reflector and as a director respectively for set and reset states of the switch. This phenomenon could be explained with reference to the characteristics of the MIM switch reported in [4]. A MIM switch could be modelled as an RC parallel network [4] as shown in inset of Fig 1. 


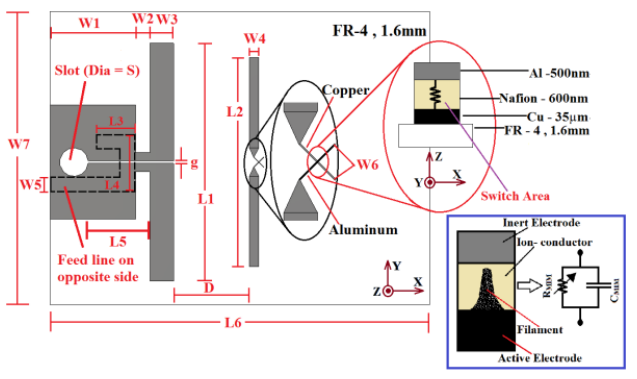

Fig. 1. Geometry of pattern-reconfigurable antenna. Inset: equivalent circuit and layer architecture of MIM switch. (W1 $=18 \mathrm{~mm}, \mathrm{~W} 2=\mathrm{W} 5=3 \mathrm{~mm}$, $\mathrm{W} 3=5 \mathrm{~mm}, \mathrm{~W} 4=2 \mathrm{~mm}, \mathrm{~W} 6=0.1 \mathrm{~mm}, \mathrm{~W} 7=60 \mathrm{~mm}, \mathrm{~L} 1=50 \mathrm{~mm}, \mathrm{~L} 2=44 \mathrm{~mm}$, $\mathrm{L} 3=8 \mathrm{~mm}, \mathrm{~L} 4=12 \mathrm{~mm}, \mathrm{~L} 5=13 \mathrm{~mm}, \mathrm{~L} 6=80 \mathrm{~mm}, \mathrm{D}=16 \mathrm{~mm}, \mathrm{~s}=6 \mathrm{~mm}, \mathrm{~g}=0.5 \mathrm{~mm})$

During set state of the switch, filament resistance $\mathrm{R}_{\mathrm{MIM}}$ is a very low value and bypasses the current path through $\mathrm{C}_{\mathrm{MIM}}$, neglecting it from current path, allowing the PE to act as a classic reflector. Conversely during reset state, $\mathrm{R}_{\mathrm{MIM}}$ is a large value and most of the current flows through $\mathrm{C}_{\mathrm{MIM}}$, thereby reducing the effective electrical length of $\mathrm{PE}$, forcing it to act as a director element. Photograph of the fabricated antenna, along with the microphotograph of MIM switch area is depicted in Fig. 2.

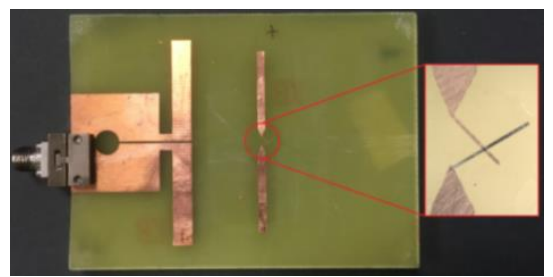

Fig. 2. Photograph of fabricated reconfigurable antenna showing the microphotograph of the switch area in the inset.

\section{RESULTS AND DISCUSSIONS}

Experimental results of the proposed dipole antenna (insertion loss characteristics and radiation pattern), are depicted in Fig. 3 and 4 respectively. One could observe that we have a usable $-10 \mathrm{~dB}$ bandwidth of $54 \mathrm{MHz}$ in common for both set and reset states as shown in Fig. 3 .

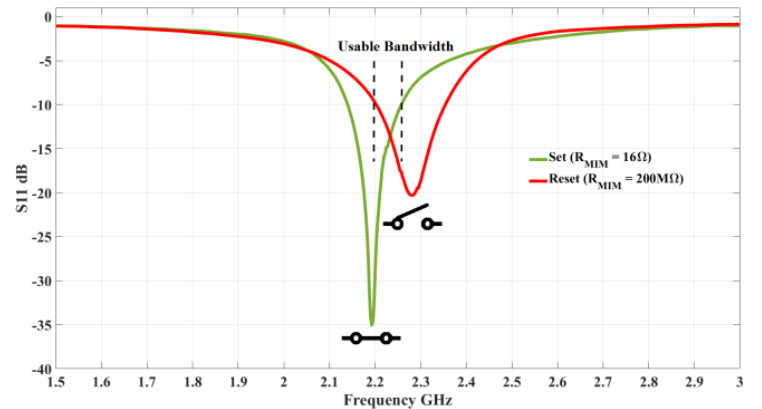

Fig. 3. S11 (Experiment) of proposed reconfigurable antenna.

Directivity patterns at $2.23 \mathrm{GHz}$ (which is the intersection of set and reset state $\mathrm{S} 11$ ) normalized to the maximum value of major lobe of reset state, are presented in Fig.4. A steering of $180^{\circ}$ is attained for the major lobe, in the plane perpendicular (along $\mathrm{X}$-direction in Fig.1) to the axis of dipole, for set and reset states of the switch. H-plane pattern shows a beam maxima towards positive $\mathrm{X}$ direction, of magnitude $0 \mathrm{~dB}$ (normalized maxima) with a back-lobe magnitude of $-8 \mathrm{~dB}$ for reset state and this pattern is steered by $180^{\circ}$, along negative $\mathrm{X}$ direction, to exhibit a maxima of $-3 \mathrm{~dB}$ and a back-lobe magnitude of $5.4 \mathrm{~dB}$ for set state. A similar behaviour with a difference greater than $-3.5 \mathrm{~dB}$, respectively between front and back lobes is observed for the E-Plane pattern. In total, we could say that the 3-D pattern exhibits a steering of $180^{\circ}$ from the positive to negative end-fire directions of the antenna by switching the integrated MIM cell.

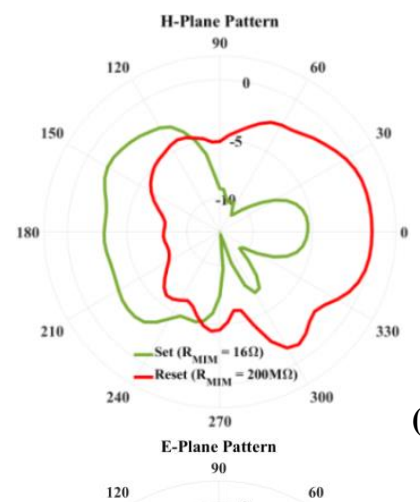

(a)

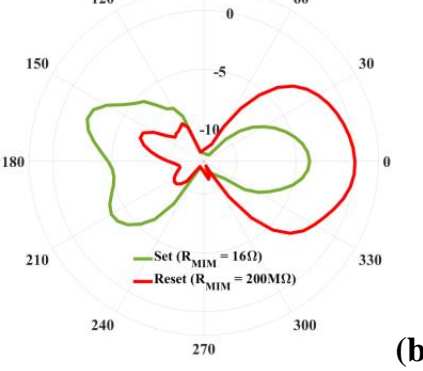

Fig. 4. Radiation patterns of the proposed antenna (a) H-Plane, (b) E-Plane.

This experiment proves the feasibility of using MIM switches for reconfigurable antenna applications. Previous experience, gives us confidence that this device could be printed without any soldered components on flexible substrates like paper along with the switch [5]. We are also extensively engaged in optimizing further and in electrical equivalent modelling of the antenna.

\section{REFERENCE}

[1] S. L. Chen et al., "Pattern Reconfigurable Antenna with Five Switchable Beams in Elevation Plane," IEEE Antennas Wirel. Propag. Lett., vol. PP, no. 99, pp. 1-1, 2018.

[2] X. Ding and B. Z. Wang, "A Novel Wideband Antenna With Reconfigurable Broadside and Endfire Patterns," IEEE Antennas Wirel. Propag. Lett., vol. 12, pp. 995-998, 2013.

[3] S. Pi et al., "Nanoscale memristive radiofrequency switches," Nat. Commun., vol. 6, p. 7519, Jun. 2015.

[4] M. P. Jayakrishnan et al., "Nafion-Based Fully Passive Solid-State Conductive Bridging RF Switch," IEEE Microw. Wirel. Compon. Lett., vol. 27, no. 12, pp. 1104-1106, Dec. 2017.

[5] J. Methapettyparambu Purushothama et al., "Electronically ReConfigurable, Non-Volatile, Nano-Ionics-Based RF-Switch on Paper Substrate for Chipless RFID Applications," Technologies, vol. 6, no. 3, p. 58, Jun. 2018.

[6] R. Li et al., "Equivalent-Circuit Analysis of a Broadband Printed Dipole With Adjusted Integrated Balun and an Array for Base Station Applications," IEEE Trans. Antennas Propag., vol. 57, no. 7, pp. 21802184, Jul. 2009. 